\title{
A Soft Wearable Robot for Tremor Assessment and Suppression
}

\author{
J.A. Gallego, E. Rocon, J. Ibáñez, J.L. Dideriksen, A.D. Koutsou, R. Paradiso, M.B. Popovic, \\ J.M. Belda-Lois, F. Gianfelici, D. Farina, D.B. Popovic, M. Manto, T. D’Alessio, and J.L. Pons
}

\begin{abstract}
Tremor constitutes the most common motor disorder, and poses a functional problem to a large number of patients. Despite of the considerable experience in tremor management, current treatment based on drugs or surgery does not attain an effective attenuation in $25 \%$ of patients, motivating the need for research in new therapeutic alternatives. In this context, this paper presents the concept design, development, and preliminary validation of a soft wearable robot for tremor assessment and suppression. The TREMOR neurorobot comprises a Brain Neural Computer Interface that monitors the whole neuromusculoskeletal system, aiming at characterizing both voluntary movement and tremor, and a Functional Electrical Stimulation system that compensates for tremulous movements without impeding the user perform functional tasks. First results demonstrate the performance of the TREMOR neurorobot as a novel means of assessing and attenuating pathological tremors.
\end{abstract}

\section{INTRODUCTION}

Tremor is defined as a rhythmic activity of a body part. Although we all exhibit a certain degree of tremor -the so called physiological tremor-, there are several pathologies that have associated very disabling tremors. This pathological tremors constitute the most common motor disorder, affecting $15 \%$ of people with ages ranging between 50 and 69 years [1]. Moreover, among people suffering from upper limb tremors, $65 \%$ of them report severe disability to perform their activities of daily living [2]. In addition to that, the mechanisms underlying the different forms of tremor are yet not understood, which often leads to misdiagnosis and subsequent treatment problems [3].

Tremor is typically managed with drugs, and with surgery (gamma knife thalamotomy) or implantation of a Deep Brain

The work presented in this paper has been carried out with the financial support from the Commission of the European Union, within Framework 7, specific IST programme "Accessible and Inclusive ICT", Target outcome 7.2 "Advanced self-adaptive ICT-enabled assistive systems based on noninvasive Brain to Computer Interaction (BCI)", under Grant Agreement number ICT-2007-224051, "TREMOR: An ambulatory BCI-driven tremor suppression system based on functional electrical stimulation."

J.A. Gallego, E. Rocon, J. Ibáñez, A.D. Koutsou, and J.L. Pons are with the Bioengineering Group, Consejo Superior de Investigaciones Científicas, CSIC, Spain, e-mail: gallego@iai.csic.es

J.L. Dideriksen, F. Gianfelici, and D.B. Popovic are with the Center for Sensory-Motor Interaction, Department of Health Science and Technology, Aalborg University, Denmark.

R. Paradiso is with SMARTEX s.r.l, Italy.

M.B. Popovic is with UNA Systems, Serbia.

J.M. Belda-Lois is with Instituto de Biomecánica de Valencia, Spain.

D. Farina is with Dept. Neurorehab Eng, Bernstein Center for Computational Neuroscience, Georg-August University, Göttingen, Germany.

D.B. Popovic also is with Faculty of Electrical Engineering, University of Belgrade, Serbia.

M. Manto is with Service de Neurologie, Hôpital Erasme-ULB, Bruxelles, Belgium.

T. D'Alessio is with Biolab, University of Rome TRE, Rome, Italy.
Stimulator (DBS) in those patients refractory to medication. However, the drugs used often induce side effects, or may be contraindicated, and surgery is associated with a risk of hemorrhage and psychiatric manifestations, [4]. This causes that around $25 \%$ of patients suffering from pathological tremor do not benefit from an effective reduction of their symptoms, and motivates research in alternative means of compensating for tremors.

In this context, the authors validated, both functionally and clinically, tremor suppression based on the application of biomechanical loads with an upper limb robotic exoskeleton, [5]. This work relied on the known effects of impedance modulation in tremor, [6], and provided with up to $80 \%$ tremor attenuation in severe cases, without impeding the user to perform functional tasks. Nevertheless, patients were reluctant to use such an anesthetic and bulky solution as a robotic exoskeleton.

Here we present the design, development, and first results of a novel soft wearable robot for tremor assessment and suppression based on biomechanical loading. The TREMOR neurorobot monitors the neuromusculoskeletal system to characterize both concomitant voluntary and tremulous movements based on a Brain Neural Computer Interface (BNCI), and then stimulates upper limb muscles to compensate functionally for the tremor. The TREMOR neurorobot is implemented as an active garment, fulfilling users' expectances in terms of comfort and cosmetics, and incorporates arrays of sewn electrodes to apply selective biomechanical loads through Functional Electrical Stimulation (FES). Notice that attenuation of writs tremors based on muscle stimulation has been previously evaluated in [7], demonstrating the feasibility of the approach.

This paper is organized as follows. First, we review the design of the neurorobot and its development, together with a brief overview of the different experimental protocols employed in tests with users. Afterwards, we describe both the cognitive and physical human-robot interaction, summarizing the BNCI algorithms for characterization of voluntary and tremulous movements, and the control strategies to compensate for the tremor. Next, we provide first experimental results showing the performance of the different components. The paper ends with a discussion of the current results obtained, and an outline of current research.

\section{Concept Design for the Soft Wearable Robot}

The TREMOR neurorobot aims at functionally compensating for upper limb tremors, hence it incorporates sensors and actuators to control the next degrees of freedom: 1) elbow 


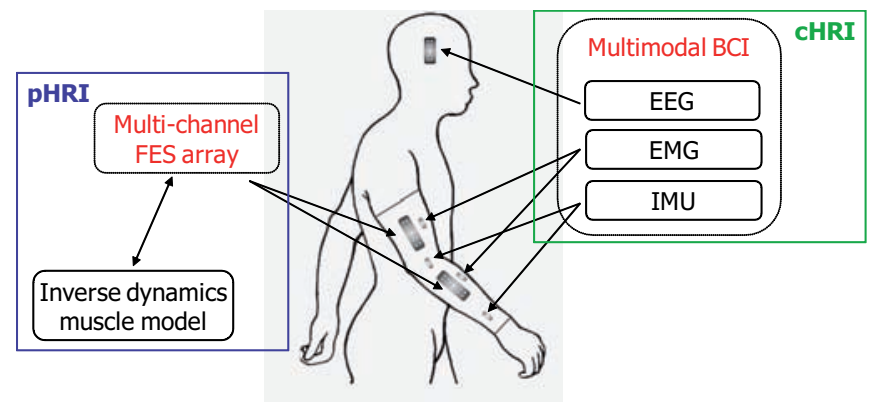

Fig. 1. Concept design of the TREMOR neurorobot, showing both physical and cognitive interfaces (pHRI and cHRI respectively).

flexion/extension, 2) forearm pronation/supination, 3) wrist flexion/extension, and 4) wrist abduction/adduction. Previous studies, [8], indicate that the former three have the largest impact on disability, thus they constitute our main focus.

As all wearable robots, the major characteristic of the TREMOR neurorobot is its strong interaction with the user, [9]. This interaction is both physical and cognitive, and happens in a bidirectional manner. In our case, the cognitive Human-Robot Interface (cHRI) is built upon a BNCI that assesses the generation, transmission, and execution of both voluntary and tremorous movements. On the other hand, the physical Human-Robot Interface (pHRI) comprises a multichannel FES system that selectively drives the muscles based on the output of the control algorithm, Fig. 1. The TREMOR neurorobot takes the shape of an active garment (a sleeve) that incorporates arrays of sewn electrodes for both electrical stimulation and recording, [10], satisfying users aesthetical' preferences and usability requirements. Moreover, stimulation through electrode matrices allows us to implement techniques to minimize fatigue, discomfort and painful sensations, [11], at the same time that we enhance the selectivity of the simulation.

The BNCI comprises recording of electroencephalographic (EEG) and electromyographic (EMG) activity, together with motion capture with inertial measurement units (IMUs). Each sensor modality aims at extracting certain information, following a hierarchical integration scheme. First, a real-time EEG classifier is in charge of detecting user's intention to perform a voluntary movement, triggering the system. Next, processing of sEMG information yields tremor onset and an estimation of its frequency and phase. Finally, IMUs track instantaneous tremor amplitude and frequency at each joint. The use of multiple sensor modalities also permits us implementing fusion and redundancy techniques to enhance the dependability of the system. One example of redundancy is the use of EMG activity to detect the occurence of a motor command, compensating for eventual EEG classification errors. An example of sensor fusion is the use of machine learning techniques to adjust the parameters of the EEG clasisifier after the execution of a movement, as detected with IMUs. Another interesting example of integration of different modalities is employing sEMG detection of tremor to feed the controller in real-time with which muscles are responsible for the tremorous movement, while IMUs are used to precisely characterize tremor features at a joint level. Identification of the muscles that are contributing to the tremulous movement with sEMG is crucial for subsequent compensation with FES; therefore adequate modulation of the stimulation cannot be attained with IMUs alone.

The physical interface of the TREMOR neurorobot aims at taking advantage of biomechanical loading as a selective means for compensating for tremor. Hence, the system will filter out the tremor, leaving concomitant voluntary motion unaltered, based on the application of FES through the array of sewn electrodes. Command signals to drive the stimulators are provided by a control strategy that relies on the information of the BNCI described above, and on an inverse dynamic model of the musculoskeletal system. We are currently evaluating two tremor suppression strategies: one based on impedance control, the other on learning control techniques, [5]. Notice that these strategies are implemented independently in every joint because tremors are known to be local phenomena, and prone to migrate to proximal joints when being mechanically atenuated, [12].

\section{Methods}

The soft wearable robot described above has been implemented as an experimental platform that comprises both the BNCI and the multichannel FES system, interfacing with the user through the active garment. A master computer running Neutrino Real-Time Operating System (QNX Software Systems, Ontario, Canada) is in charge of synchronizing and integrating the information from the different sensor modalities, and driving the FES units. This platform has been built and validated with patients in an iterative fashion, and in its current form comprises the following systems: 1) a 16 channel EEG amplifier (gUSBamp, Guger Technologies OG, Graz, Austria), 2) a 128 channel surface EMG amplifier (EMG-USB, OT Bioelettronica, Torino, Italy), 3) a motion capture system based on IMUs (TechMCS, Technaid SL, Madrid, Spain), 4) multichannel, individually controllable, electrical stimulators (Tremuna, UNA Systems, Belgrade, Serbia), and 5) an e-textile (SMARTEX, Prato, Italy). Fig. 2 shows a control subject wearing the TREMOR neurorobot.

The first results presented in this paper correspond to different experimental sessions carried out in the Erasmus Hospital (Brussels, Belgium) and in the General Hospital of Valencia (Valencia, Spain), and were collected following three protocols. The first protocol aimed at identifying and characterizing the different neurophysiological phenomena associated to movement preparation in the case of tremor patients. The second protocol was designed to activate the different types of tremor based on well described clinical tasks, so that the BNCI-based algorithms parameterize its features. The last protocol aimed at evaluating tremor suppression strategies based on FES. Between both locations, 17 patients suffering from the most common types of tremor have participated in this study, after giving written informed consent. The group of patients comprises the three major types of pathological tremor, namely Parkinsonian patients, 


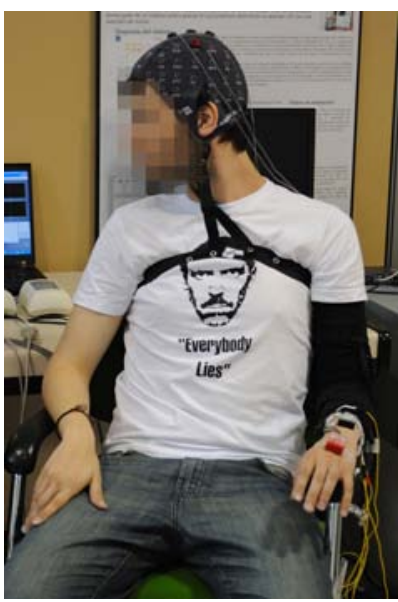

(a)

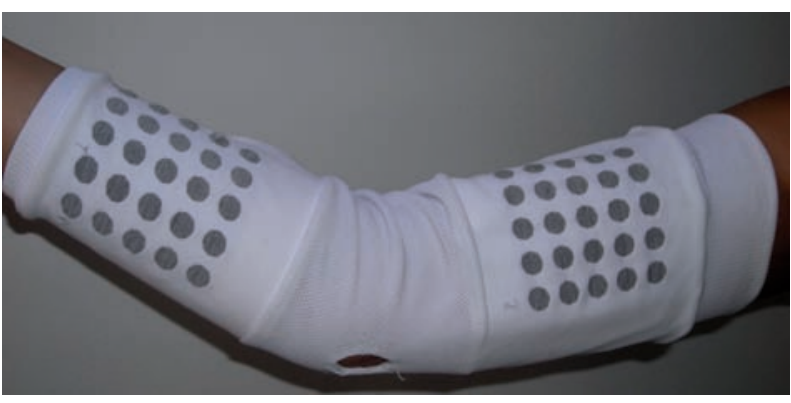

(b)

Fig. 2. (a) A control subject wearing the TREMOR neurorobot, including the EEG system and the active garment with IMUs. (b) Detail of the inner part of the active garment showing the stimulation and recording sewn electrodes.

essential tremor patients, and patients suffering from cerebellar disorders. Experimental protocols were approved by the Ethical Commitees of both hospitals.

\section{Cognitive Human-Robot Interaction}

This section describes briefly the different algorithms implemented in the BNCI to characterize both voluntary and tremorous movements.

\section{A. Detection of Movement Intention}

Detection of movement intention is carried out based on a BCI-switch that anticipates the execution of self initiated volitional movements in the special case of tremor patients. The proposed algorithm evaluates desynchronization in the alpha and beta bands at the motor cortex during movement planification and execution. This neurophysiologic phenomenom is known as Event Related Desynchronization (ERD), [13], and is vastly described on the literature, although, to the authors' knowledge, has never been systematically analyzed for tremor patients. Fig 3 shows an example of ERD in an essential tremor patient. Movement starts at time 0, and ERD is evident in the 12 and $22 \mathrm{~Hz}$ frequency bands already $2 \mathrm{~s}$ before the motion is executed.

The single trial $\mathrm{BCI}-\mathrm{switch}$ predicts movement intention by combining two Bayesian Classifiers, based on the next ERD features: 1) power in some frequency bands decreases some seconds before the movement is executed, and 2) this decay tends to be stable during a certain time interval, [13]. Both classifiers take as input the spatially filtered signal -by means of a Laplacian filter- at the three preferred positions, $\mathrm{C} 3, \mathrm{Cz}$ and $\mathrm{C} 4$.

\section{B. Detection of Tremor Onset}

Analysis of sEMG information serves to detect the onset of tremor, and gives a first estimate of its frequency. Application of the Iterated Hilbert Transform (IHT), a multicomponent

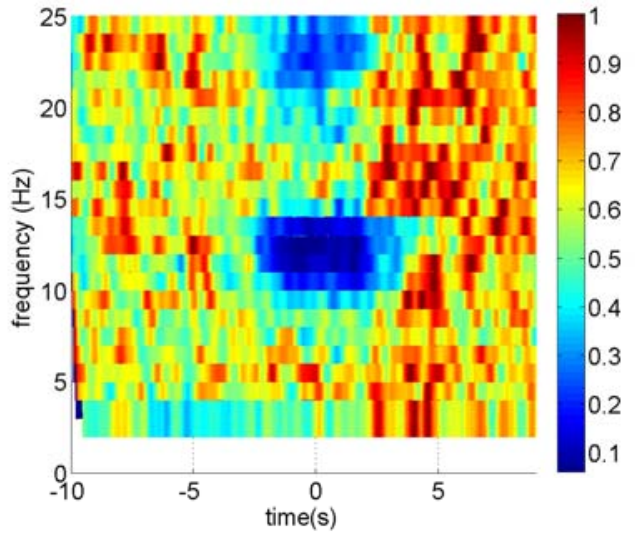

Fig. 3. Desynchronization map for an essential tremor patient. The ERD map has been obtained after spatial filtering of the EEG signal around the contralateral motor cortex. Cold colours correspond to low power.

AM-FM decomposition method [14] on the raw sEMG signal serves to extract the tremorours component from the voluntary activity component, without any a priori information about tremor frequency, its spectrum, and its nonstationary dynamics. Moreover, as the correlation between the tremor component and the oscillatory movement is significantly high, this algorithm lets us obtain an estimate of tremor frequency.

\section{Estimation of Tremor Features}

The algorithm to estimate instantaneous tremor features at every joint first obtains joint rotation by differential measurement with two IMUs placed distally and proximally with respect to it. Then it estimates, from the raw motion, the volitional and tremulous components of the rotation. Voluntary movement and tremor are separated based on the fact that they occur in different frequency bands, and can be considered to be additive, [15].

Volitional movement is then modelled as a first order 


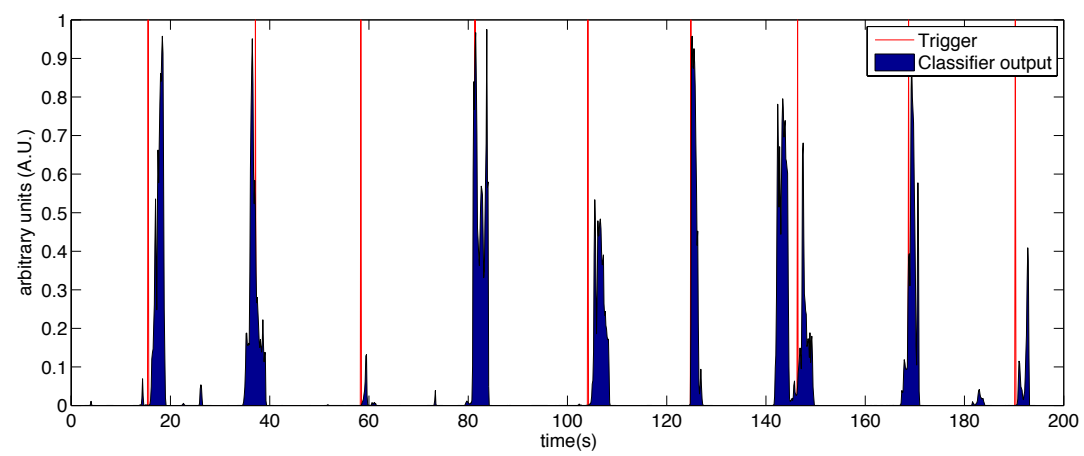

Fig. 4. Example of detection of movement intention with the proposed algorithm in an essential tremor patient. The blue surface corresponds to the raw combined output of both classifiers, red lines to the execution of movements.

process that is estimated by a Critically Dampened Filter, a special type of $\mathrm{g}-\mathrm{h}$ filter. By removing the estimated voluntary component from the raw motion, we obtain an estimation of the tremor. This estimation of tremor is then fed into two adaptive algorithms. The first algorithm is the Weighted Frequency Fourier Linear Combiner (WFLC), which is employed to track tremor frequency based on a LMS approach, [16]. Afterwards, a Kalman Filter that incorporates a harmonic model of the process, estimates tremor amplitude taking WFLC frequency estimation into account.

\section{Physical Human-Robot Interaction}

Due to the differences among symptoms in the different types of tremor, we are evaluating two control strategies for the selective application of biomechanical loading through FES. The first of them is based on modification of the apparent impedance of the limb, whereas the second one relates to noise canceling techniques. The reason for this is two fold. On the one hand, a previous study suggests that the latter strategy is more efficient, although it was only compared with addition of constant -nonmodulatedviscosity, [5]. On the other hand, even with modulated impedance, as Parkinson's disease carries increased limb rigidity, we believe that a noise cancelling approach will still yield more tremor attenuation for these patients. Both strategies are described next.

The impedance modulation strategy relies on modifying the apparent joint stiffness and viscosity so that tremor is naturally filtered out, leaving concomitant voluntary movement unaffected. Considering that a human joint may be simply modelled as a second order system, [17], increased rigidity or damping decreases the cut off frequency of the low pass filter response of the joint, hence allowing for tremor attenuation. This is similar to the cocontraction strategy employed by healthy subjects to stabilize the upper limb in a variety of situations, [18].

The noise canceling like approach generates an oscillation with the same amplitude but opposite phase to the tremor, compensating for it. This strategy is implemented as a repetitive controller which memory block is adapted following the instantaneous estimation of tremor frequency. According to learning control theory, if the prediction of perturbation frequency is accurate enough, the system will reach to zero steady state error, [19]. Nevertheless, as tremor is a dynamic phenomenon, we correct the prediction of tremor frequency based on the instantaneous amplitude, as it indicates which muscle to stimulate.
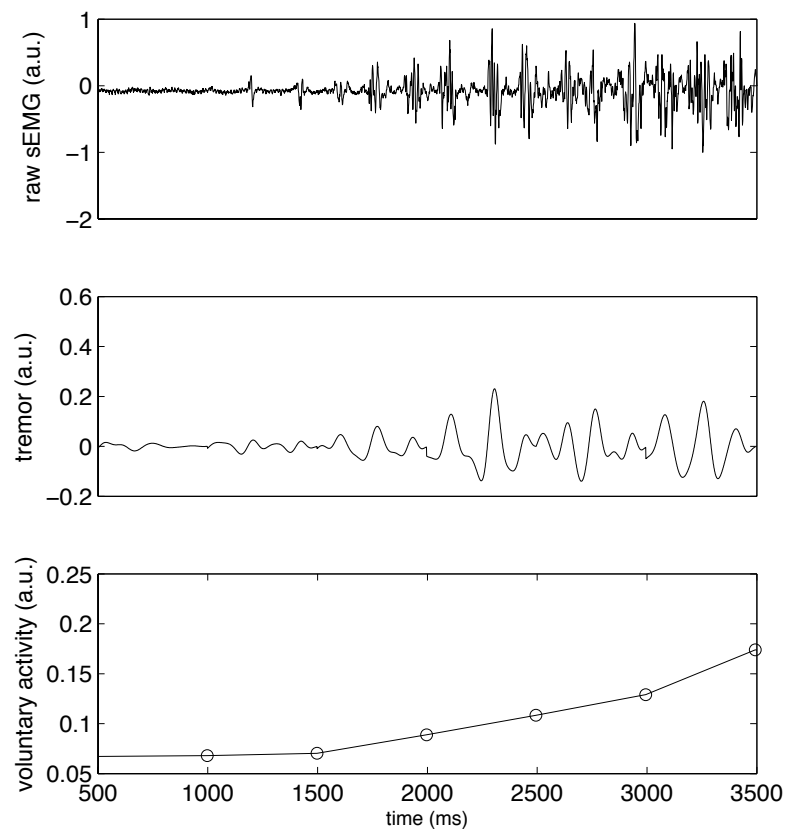

Fig. 5. Decomposition of tremor recorded with surface EMG electrodes Top panel shows the raw EMG signal. Middle and bottom panels show the estimation of tremor and voluntary activity obtained with the IHT algorithm.

\section{First Results}

This section presents results obtained with the BNCI algorithms and the tremor suppression strategies presented above.

\section{A. Detection of Movement Intention}

The performance of the EEG classifier is evaluated following an event like approach, thus the output of the classifier 
is compared with a certain threshold to translate the probabilistic output of the Bayessian classifiers to a binary signal. The figures of merit employed are the precision and recall.

Fig. 4 shows an example of detection of movement intention in a tremor patient. The figure shows the raw combined output of both classifiers, and the execution of movements. Results with 4 tremor patients suffering from different pathologies yield a precision of $46.5 \pm 10.66 \%$, and a recall of $69.5 \pm 14.76 \%$. Average anticipation of the Bayesian classifier is $320 \pm 141 \mathrm{~ms}$, proving the ability of the proposed algorithm to predict the execution of a voluntary movement, [20].

\section{B. Detection of Tremor Onset}

Extraction of tremor information from sEMG has been validated with a novel sEMG tremor model [21] and with data from tremor patients. The first analysis serves to infer a series of relationships that cannot be obtained from real data, such as the correlation of the tremor estimation with the central tremor oscillator, and the amount of voluntary muscle contraction. Performance of the proposed algorithm with patients data is evaluated by computing the crosscorrelation with the information of tremor provided by the IMUs, and the phase advance of the sEMG tremor estimation with respect to movement.

Model results show significant correlation between the imposed voluntary activation and that estimated with the IHT, and also between imposed tremor intensity and its estimation. Results with signals collected from 4 tremor patients yield a crosscorrelation of $0.62 \pm 0.15$, and a phase advance of $13.0 \pm 9.9 \mathrm{~ms}$. Fig. 5 shows an example of detection of tremor from sEMG. The proposed algorithm provides not only tremor onset, but also an estimation of its shape, and the voluntary activity exerted by the muscle.

\section{Estimation of Tremor Features}

Both stages of the algorithm to estimate tremor parameters have been evaluated stepwise. First, voluntary movement tracking is assessed using the Kinematic Tracking Error (KTE), a figure of merit that accounts for the estimation error and the variance of the estimator, [22]. Next, the performance of the tremor estimation algorithm is evaluated by computing the Filtered Mean Square Error with Delay Correction $\left(\mathrm{FMSE}_{\mathrm{d}}\right)$, a metric specifically proposed for tremor tracking filters, [23]. Estimation of frequency estimation is validated by comparing it with the spectrogram.

Analysis of data from five patients suffering from tremor with different aetiologies, provides with a KTE of $0.223 \pm$ $0.082 \mathrm{rad} / \mathrm{s}$, and a $\mathrm{FMSE}_{\mathrm{d}}$ of $0.001 \pm 0.002 \mathrm{rad} / \mathrm{s}$, [22]. Fig. 6 shows an example of estimation of voluntary movement, instantaneous tremor amplitude and frequency in a parkinsonian patient performing a postural test. Accurate tracking is obtained.

\section{Attenuation of Tremor}

Validation of tremor suppression strategies is being carried out in an iterative fashion. First, we have evaluated tolerance
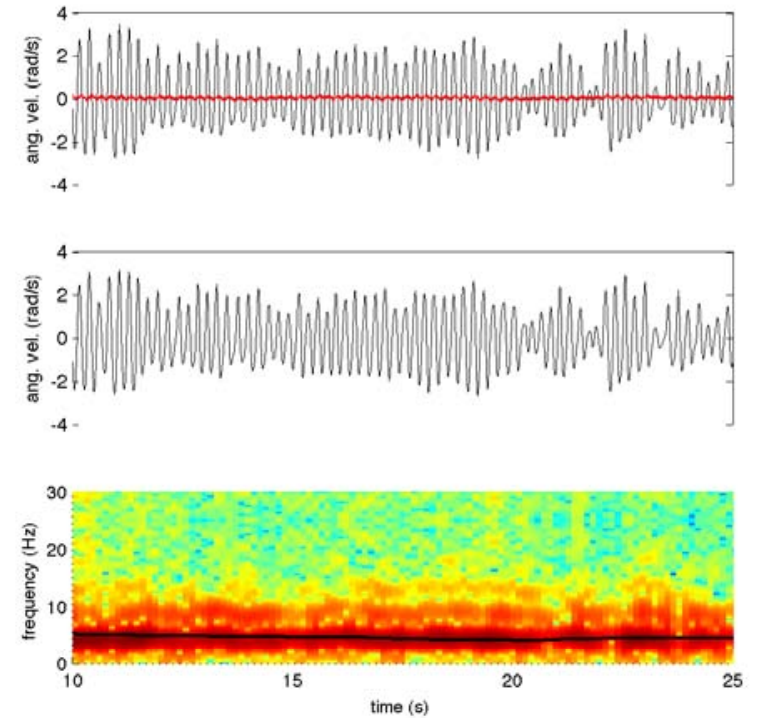

Fig. 6. Estimation of instantaneous tremor parameters during a postural task performed by a parkinsonian patient. Top panel shows total movement (black) and the estimation of voluntary movement (red). Middle and bottom panels show estimation of tremor amplitude and frequency (overimposed to the movement spectrogram) respectively.

of tremor patients to FES in terms of discomfort and pain. Afterwards, we have studied the role that muscle FES induced fatigue plays in tremorogenic muscles. Next we have tested the impedance control strategy in open loop, i.e. with constant stiffness and damping increase. Current work focuses on evaluation of both strategies in closed loop.

Preliminary results indicate that, as expected, results vary considerably among patient groups. First trials suggest that modification of joint impedance is effective in the suppression of tremor. Fig. 7 shows attenuation of wrist tremor in an essential tremor patient while performing a postural task. In this trial, tremor attenuation reaches approximately $60 \%$ of the peak to peak value. Experimental results show migration of tremor to proximal joints when tremor is cancelled out through FES, supporting the need of independent joint controllers.

\section{DISCUSSION}

Previous sections presented the design, and development of the TREMOR neurorobot, a novel soft wearable robot that aims at characterizing and suppressing for upper limb tremors with different aetiology.

First results demonstrate the ability of the cHRI to predict user's intention to perform a volitional movement, to detect the presence of tremor from sEMG, and to estimate its instantaneous amplitude and frequency out of kinematic information. Moreover, we are integrating a number of features that will serve to enhance the reliability of the neurorobot, for example: 1) taking advantage of the sEMG algorithm to estimate voluntary movement activity to compensate for BCI based classification errors, 2) using the frequency estimation 


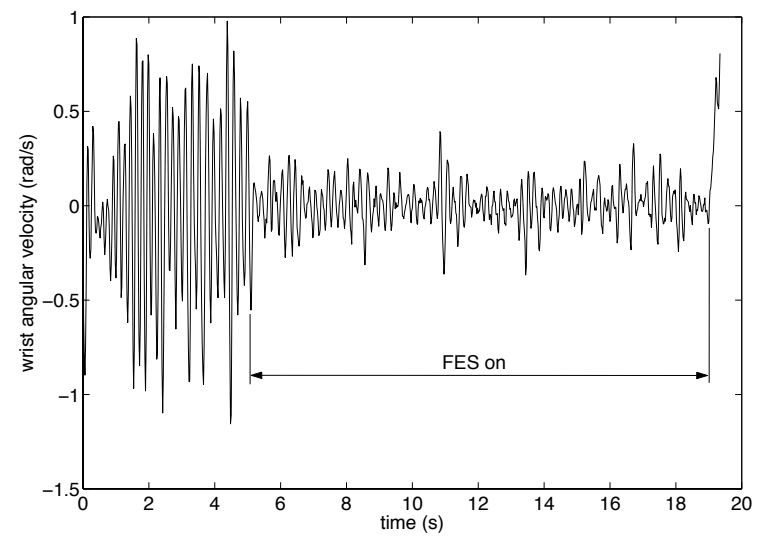

Fig. 7. Compensation of tremor in the case of an essential tremor patient keeping his arms outstrechted. During FES a considerable attenuation is observed, although tremor reappears when stimulation ceases.

obtained by the IHT algorithm as an initial guess for the IMU based algorithm to track tremor features, 3) implementing machine learning techniques to adjust the parameters of the Bayesian classifier online, based on the execution of a voluntary movement.

Regarding FES based tremor suppression, though the results are yet preliminary, we can draw a number of conclusions from them. First, impedance modulation is mandatory to attain a significant degree of tremor attenuation. In this regard, we are working on a model based adaptive controller to estimate the apparent impedance of the joint. Other approaches based in impedance adaptation of the muscular system will also be explored, [24]. Second, determining which muscles contribute to joint tremor (from sEMG) is mandatory for efficient cancellation with FES.

\section{CONCLUSIONS}

This paper presents the concept design, development, and preliminary validation of a soft wearable robot for tremor assessment and suppression. The TREMOR neurorobot comprises a cHRI based on a BNCI that monitors the whole neuromusculoskeletal system, aiming at characterizing both voluntary movement and tremor, and a pHRI that relies on FES to compensate for tremulous movement without impeding the user's functional movements. The soft robot interfaces with the user through sewn electrodes incorporated in an active garment, which takes the shape of a sleeve, thus aimed at satisfying users' aesthetic and cosmetic preferences. First results with patients suffering from different types of tremor, show the ability of the TREMOR neurorobot to model the generation, transmission, and execution of both volitional and concomitant tremulous movements. Moreover, these tests demonstrate the ability of the system to attain considerable attenuation of tremors in some patients.

\section{REFERENCES}

[1] G.K. Wenning, S. Kiechl, K. Seppi, J. Müller, J. Högl, M. Saletu, G. Rungger, A. Gasperi, J. Willeit, and W. Poewe, "Prevalence of movement disorders in men and women aged 50-89 years (bruneck study cohort): A population-based study," Lancet Neurol, vol. 4, no. 12 , pp. $815-820,2005$.

[2] E. Rocon, J. M. Belda-Lois, J. M. Sanchez-Lacuesta, and J. L. Pons, "Pathological tremor management: Modelling, compensatory technology and evaluation," Tech Disab, vol. 16, pp. 3-18, 2004.

[3] E. D. Louis, "Essential tremors: a family of neurodegenerative disorders?" Arch Neurol, vol. 66, no. 10, pp. 1202-1208, 2009.

[4] D. K. Binder, G. Rau, and P. A. Starr, "Hemorrhagic complications of microelectrode-guided deep brain stimulation," Stereotact Funct Neurosurg, vol. 30, pp. 28-31, 2003.

[5] E. Rocon, J. M. Belda-Lois, A. F. Ruiz, M. Manto, J. C. Moreno, and J. L. Pons, "Design and validation of a rehabilitation robotic exoskeleton for tremor assessment and suppression," IEEE Tans Neural Syst Rehab Eng, vol. 15, no. 3, pp. 367-378, 2007.

[6] G. C. Joyce and P. M. H. Rack, "The effects of load and force on tremor at the normal human elbow joint," $J$ Physiol, vol. 240, pp. 375-396, 1974.

[7] A. Prochazka, J. Elek, and M. Javidan, "Attenuation of pathological tremors by functional electrical stimulation. i: Method." Ann Biomed Eng, vol. 20, no. 2, pp. 205-224, 1992.

[8] J. M. Belda-Lois, E. Rocon, J. J. Sánchez-Lacuesta, A. F. Ruiz, and J. L. Pons, "Functional assessment of tremor in the upper limb," in Euro Conf Advancement Assistive Techn in Europe, 2005.

[9] J. L. Pons, Ed., Wearable Robots: Biomechatronic Exoskeletons. John Wiley \& Sons, Ltd, 2008.

[10] R. Paradiso, L. Caldani, M. Pacelli, F. Negro, and D. Farina, "E-textile platforms for rehabilitation," in Conf Proc IEEE Eng Med Biol Soc, 2010.

[11] A. Popovic-Bijelic, G. Bijelic, N. Jorgovanovic, D. Bojanic, M. B. Popovic, and D. B. Popovic, "Multi-field surface electrode for selective electrical stimulation." Artif Organs, vol. 29, no. 6, pp. 448-452, 2005. [Online]. Available: http://dx.doi.org/10.1111/j.15251594.2005.29075.x

[12] M. Manto, E. Rocon, J. L. Pons, J. M. Belda-Lois, and S. Camut, "Evaluation of a wearable orthosis and an associated algorithm for tremor suppression." Physiol Meas, vol. 28, no. 4, pp. 415-425, 2007. [Online]. Available: http://dx.doi.org/10.1088/0967-3334/28/4/007

[13] G. Pfurtscheller and F. H. Lopes da Silva, "Event-related eeg/meg synchronization and desynchronization: basic principles," Clin Neurophysiol, vol. 110, no. 11, pp. 1842 - 1857, 1999. [Online]. Available: http://www.sciencedirect.com/science/article/B6VNP3XJSSG7-1/2/919fb705601eee998c08fc2160dc2f99

[14] F. Gianfelici, G. Biagetti, P. Crippa, and C. Turchetti, "Multicomponent am-fm representations: An asymptotically exact approach," IEEE Trans Audio Speech Lang Process, vol. 15, no. 3, pp. 823-837, 2007.

[15] P. O. Riley and M. J. Rosen, "Evaluating manual control devices for those with tremor disability," J Rehabil Res Dev, vol. 24, no. 2, pp. 99-110, 1987.

[16] C. N. Riviere, R. S. Rader, and N. V. Thakor, "Adaptive canceling of physiological tremor for improved precision in microsurgery," IEEE Trans Biomed Eng, vol. 45, no. 7, pp. 839-846, 1998.

[17] E. Rocon, R. A. F., and P. J. L., "Biomechanical modelling of the upper limb for robotics-based orthotic tremor suppression," Appl Bionics Biomech, vol. 2, no. 2, pp. 81-85, 2005.

[18] N. Hogan, "Adaptive control of mechanical impedance by coactivation of antagonist muscles," IEEE Trans Autom Control, vol. 29, no. 8, pp. $681-690$, aug 1984.

[19] S. Hara, Y. Yamamoto, T. Omata, and M. Nakano, "Repetitive control system: a new type servo system for periodic exogenous signals," IEEE Trans Autom Control, vol. 33, no. 7, pp. 659 -668, jul 1988.

[20] J. Ibáez, J. I. Serrano, M. del Castillo, and L. Barrios, "An asynchronous bmi system for online single-trial detection of movement intention," in Conf Proc IEEE Eng Med Biol Soc, 2010.

[21] J. L. Dideriksen and D. Farina, "An integrative model of the surface emg in pathological tremor," in Conf Proc IEEE Eng Med Biol Soc, 2010.

[22] J. A. Gallego, E. Rocon, J. O. Roa, J. C. Moreno, and J. L. Pons, "Realtime estimation of pathological tremor parameters from gyroscope data," Sensors, vol. 10, no. 3, pp. 2129-2149, 2010.

[23] J. G. Gonzalez, E. A. Heredia, T. Rahman, K. E. Barner, and G. R. Arce, "Optimal digital filtering for tremor suppression," IEEE Trans Biomed Eng, vol. 47, no. 5, pp. 664-673, 2000.

[24] K. P. Tee, D. W. Franklin, T. Milner, M. Kawato, and E. Burdet, "Concurrent adaptation of force and impedance in the redundant muscle system," Biol Cybern, vol. 102, pp. 31-44, 2010. 